\title{
NETest liquid biopsy is diagnostic of small intestine and pancreatic neuroendocrine tumors and correlates with imaging
}

\author{
Anna Malczewska', Magdalena Witkowska ${ }^{1}$, Karolina Makulik', Agnes Bocian ${ }^{1}$, Agata Walter ${ }^{1}$, \\ Joanna Pilch-Kowalczyk ${ }^{2}$, Wojciech Zajęcki ${ }^{3}$, Lisa Bodei ${ }^{4}$, Kjell Oberg ${ }^{5}$ and Beata Kos-Kudła ${ }^{1}$ \\ 'Department of Endocrinology and Neuroendocrine Tumors, Department of Pathophysiology and Endocrinology, Medical University of Silesia, \\ Katowice, Poland \\ 2Department of Radiology and Nuclear Medicine, Medical University of Silesia, Katowice, Poland \\ 3Department of Pathology in Zabrze, Medical University of Silesia, Katowice, Poland \\ ${ }^{4}$ Molecular Imaging and Therapy Service, Department of Radiology, Memorial Sloan Kettering Cancer Center, New York, New York, USA \\ ${ }^{5}$ Department of Endocrine Oncology, University Hospital, Uppsala, Sweden
}

Correspondence should be addressed to A Malczewska or K Oberg: malczewska.an@gmail.com or kjell.oberg@medsci.uu.se

\begin{abstract}
Introduction: Current monoanalyte biomarkers are ineffective in gastroenteropancreatic neuroendocrine tumors (GEP-NETs). NETest, a novel multianalyte signature, provides molecular information relevant to disease biology.

$\operatorname{Aim}(s)$ : Independently validate NETest to diagnose GEP-NETs and identify progression in a tertiary referral center.

Materials and methods: Cohorts are 67 pancreatic NETs (PNETs), 44 small intestine NETs (SINETs) and 63 controls. Well-differentiated (WD) PNETs, $n=62$, SINETs, all $(n=44)$. Disease extent assessment at blood draw: anatomical $(n=110)$ CT $(n=106)$, MRI $(n=7)$ and/or functional ${ }^{68} \mathrm{Ga}-\mathrm{SSA}-\mathrm{PET} / \mathrm{CT}(n=69)$ or ${ }^{18} \mathrm{~F}$-FDG-PET/CT $(n=8)$. Image-positive disease (IPD) was defined as either CT/MRI or ${ }^{68} \mathrm{Ga}-\mathrm{SSA}-\mathrm{PET} / \mathrm{CT} /{ }^{18} \mathrm{~F}$-FDG-PET/CT-positive. Both CT/MRI and ${ }^{68} \mathrm{Ga}-\mathrm{SSA}-\mathrm{PET} / \mathrm{CT}$ negative diagnosis in WD-NETs was considered imagenegative disease (IND). NETest (normal: 20): PCR (spotted plates). Data: mean \pm SD. Results: Diagnosis: NETest was significantly increased in NETs $(n=111 ; 26 \pm 21)$ vs controls $(8 \pm 4, p<0.0001)$. Seventy-five (42 PNET, 33 SINET) were image positive. Eleven (8 PNET, 3 SINET; all WD) were IND. In IPD, NETest was significantly higher (36 \pm 22$)$ vs IND $(8 \pm 7$, $P<0.0001$ ). NETest accuracy, sensitivity and specificity are 97,99 and $95 \%$, respectively. Concordance with imaging: NETest was 92\% (101/110) concordant with anatomical imaging, 94\% (65/69) with ${ }^{68} \mathrm{Ga}-\mathrm{SSA}-\mathrm{PET} / \mathrm{CT}$ and $96 \%$ (65/68) dual modality (CT/MRI and ${ }^{68} \mathrm{Ga}-\mathrm{SSA}$-PET/CT). In $70 \mathrm{CT} / \mathrm{MRI}$ positive, NETest was elevated in all (37 \pm 22$)$. In $40 \mathrm{CT} / \mathrm{MRI}$ negative, NETest was normal $(11 \pm 10)$ in 31. In $56{ }^{68} \mathrm{Ga}$-SSA-PET/CT positive, NETest was elevated (36 \pm 22$)$ in 55. In $13{ }^{68} \mathrm{Ga}-\mathrm{SSA}$-PET/CT negative, NETest was normal $(9 \pm 8)$ in ten. Disease status: NETest was significantly higher in progressive $(61 \pm 26 ; n=11)$ vs stable disease $(29 \pm 14 ; \mathrm{n}=64 ; P<0.0001)$ (RECIST 1.1). Conclusion: NETest is an effective diagnostic for PNETs and SINETs. Elevated NETest is as effective as imaging in diagnosis and accurately identifies progression.
\end{abstract}

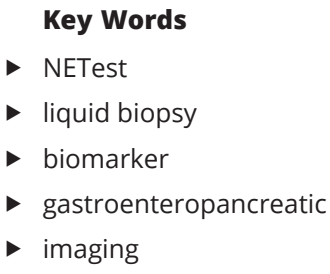

Endocrine Connections (2019) 8, 442-453

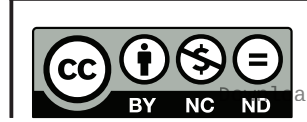

This work is licensed under a Creative Commons Attribution-NonCommercial-NoDerivatives 4.0 Internationad ticense.ifica. com at 04/26/2023 11:44:54AM 


\section{Introduction}

Neuroendocrine tumors (NETs) have increased in incidence with small intestine NETs (SINETs) being the most common and pancreatic NETs (PNETs), the third most common among gastroenteropancreatic (GEP) NETs per the SEER database (1). The increase has been attributed inter alia to improvements in tumor detection. However, current diagnostic strategies that depend on suspicious symptomatology are ineffective as $40-50 \%$ of SINETs and PNETs are not detected until distant metastases develop (2); this significantly worsens prognosis (3). The majority of PNETs (60-90\%) are non-functional and, in general, are diagnosed at a more advanced disease stage than functional (F) tumors due to the absence of 'alarming' symptoms (4). The most common F-PNETs are insulinomas. These are principally benign ( $>90 \%)$ and only $\sim 10 \%$ represent a diagnostic and therapeutic challenge $(4,5)$. SINETs are not usually clinically symptomatically active until hepatic metastasis (30-40\%) (6). In PNETs without liver involvement, carcinoid syndrome occurs very rarely (2). Circulating indicators of tumor secretory functionality (amines or peptides) hence represent a minority of NETs. Measurement of secretory biomarkers is therefore de facto clinically limited. Chromogranin A ( $\mathrm{CgA})$, previously considered the neuroendocrine pan-biomarker, reflects secretory activity rather than tumor biology, heterogeneity or plasticity $(7,8)$. It has limited clinical utility as well as methodological restrictions $(9,10)$.

Imaging remains a key component of diagnostic work-up or disease monitoring, for example, identification of disease status or therapeutic efficacy (11). RECIST and RECIST 1.1 criteria, most-widely used for the assessment of response in clinical trials, were developed based on data from testing cytotoxic drugs (12). It exhibits limitations in indolent NETs $(13,14)$ and has issues with the assessment of disease stabilization and 'pseudo-progression' (related to targeted therapy), reproducibility, lesion dimensionality evaluation or accurate delineation of metastatic disease, in particular in the extra-hepatic location. Functional molecular imaging with somatostatin receptor-based strategies for example, ${ }^{68} \mathrm{Ga}$-SSA-PET/CT, has improved the sensitivity of NET detection (15), especially in extrahepatic localization. It has benefit in the assessment of eligibility for peptide receptor radionuclide therapy (PRRT) and somatostatin analogs (SSAs). Its accuracy, however, is limited by resolution ( $\sim-8 \mathrm{~mm}$ for PET scanners), volume averaging of small lesions with adjacent normal tissues with resulting normal/low uptake, elevated background liver uptake, tumor heterogeneity or somatostatin receptor (SSTR) status and so forth. No imaging modality is exclusively effective and all are expensive and all except MRI involve radiation. The combination of anatomic and functional modalities is effective in amplifying sensitivity and specificity parameters for detection (13); this, however, increases radiation exposure.

A concern with imaging accuracy has been identified by pathological examination of liver resections from SINETs and PNETs which reveals that in $>50 \%$ (more in SINETs than PNETs), micrometastases is not detectable by various imaging modalities or macroscopic gross examination (16, 17). The incidence of occult or covert disease, especially in the liver, is therefore likely significantly higher than reported (17) and, in consequence, disease burden is underestimated. While metastatic disease represents a significant negative prognostic factor $(3,18)$, a key issue in GEP-NET management is the early identification of progressive disease at the initial stages of the metastatic cascade. As a result, there can be a time delay in the introduction of efficacious treatment. To date, no blood-based biomarker for prediction of treatment efficacy is available except the positive predictor quotient for PRRT (19).

Emerging precision medicine strategies have drawn attention to the utilization of molecular tools such as non-invasive liquid biopsies (circulating biomarkers) to facilitate and optimize cancer management in many cancers (20). Recently, a molecular NET transcriptomic analysis (NETest) has been proposed as a NET liquid biopsy (21). The NETest, as opposed to CgA or other monoanalyte peptides/hormones, is a multianalyte molecular signature representing biological information pertinent to the clinical neuroendocrine disease course. It has numerous documented applications including diagnosis, identification of residual disease post surgery, disease status identification and assessment of treatment efficacy (22). The NETest has been shown to correlate with disease positivity on imaging (23). In addition, a positive blood result can also precede the standard imaging detection of the disease by 1-2 years (24). Independent validation of this NET liquid biopsy is required. We therefore undertook to validate the NETest as a diagnostic and disease status identification marker in SINETs and PNETs under real-world conditions.

\section{Aims}

The current study independently assessed the diagnostic accuracy of the NETest in SINET and PNET and for identification of progressive versus stable disease in a 
tertiary referral center. We compared NETest results to controls, assessed the concordance between NETest levels and imaging (anatomical/functional) at the time of blood draw and evaluated its accuracy for the delineation of disease status (stable and progressive disease) as defined by RECIST 1.1 .

\section{Materials and methods}

\section{Strategy}

We examined circulating NETest levels from PNETs $(n=67)$ and SINETs $(n=44)$ and compared these with controls $(n=63)$. The diagnostic accuracy and metrics (AUROC (area under the receiver operating characteristic curve), sensitivity, specificity) for the NETest were calculated. The concordance of the NETest levels with imaging was then assessed. Disease extent was determined at blood draw by anatomical imaging $(n=110)$ : CT $(n=106)$ or MRI $(n=7)$ and/or functional $-{ }^{68} \mathrm{Ga}$-DOTA-TATE PET/CT $(n=69)$ in WD NETs or ${ }^{18}$ F-FDG-PET/CT $(n=8)$ in G2/G3 NETs (Fig. 1). Image-positive disease (IPD) was defined as either CT or MRI or ${ }^{68} \mathrm{Ga}-\mathrm{SSA} /{ }^{18} \mathrm{~F}-\mathrm{FDG}$-PET/CT positive. Imagenegative disease (IND) was anatomically (CT/MRI) and functionally ${ }^{68} \mathrm{Ga}$-SSA-PET/CT (in WD NETs) negative.

\section{Imaging}

For radiological assessment of the disease, anatomical and functional imaging modalities were utilized. Anatomical imaging comprised multiphase CT with administration of iodine contrast (in two cases, CT without contrast was performed: one due to renal failure and the second due to contrast allergy). Most were performed by the 16-slice LightSpeed CT Scanner (General Electrics, Boston, MA, USA) (the slice thickness was 1.25 and $2.5 \mathrm{~mm}$ for the examination of the pancreas and $2.5 \mathrm{~mm}$ for the bowel) or multiphase MRI. The latter were performed using the 1.5 T MRI scanner (General Electrics) with injection of the gadolinium contrast (slice thickness $4-6 \mathrm{~mm}$ ), and T1/T2 sequences were obtained. The functional imaging was performed by the hybrid PET/CT scanners (positron emission tomography combined with a computer tomography scanner) with administration of ${ }^{68} \mathrm{Ga}$-DOTA-TATE in the WD NETs or ${ }^{18}$ F-FDG in higher grade (well- and poorly differentiated) tumors.

\section{Disease status}

Progressive disease was defined based on anatomical imaging and RECIST 1.1 criteria. Parameters were at least $20 \%$ increase in the sum of diameters of the target lesions (min. $5 \mathrm{~mm}$ ) measured on anatomical imaging (mostly CT) or detection of new lesions by imaging of the same modality when subsequently performed. The time interval of follow-up was a median of 8 months (range: 1.5-24 months).

\section{Cohorts}

The study was approved by the Ethics Committee of the Medical University of Silesia. Informed written consent was obtained from all study subjects. Blood samples were collected prospectively between April 2017 and April 2018. Control subjects $(n=63)$ included family members of the hospital personnel, and non-affected family members of the patients attending the Endocrinology Department. Control subjects were enrolled if there was no known malignancy present at the time of blood draw and they identified themselves as asymptomatic and in good health. All NETs were histologically confirmed, with no other synchronous malignancy at blood draw. Patient cohorts included PNETs $(n=67)$ and SINETs $(n=44)$.

Sixty-five PNETs were sporadic, 2 MEN-1 related, 58 were non-functional (NF), while the 9 functional included 5 insulinomas and 4 subjects with carcinoid syndrome (CS). WD PNETs comprised 62/67 (in two no data). Grade: G1: 33, G2: 27, G3: 5.

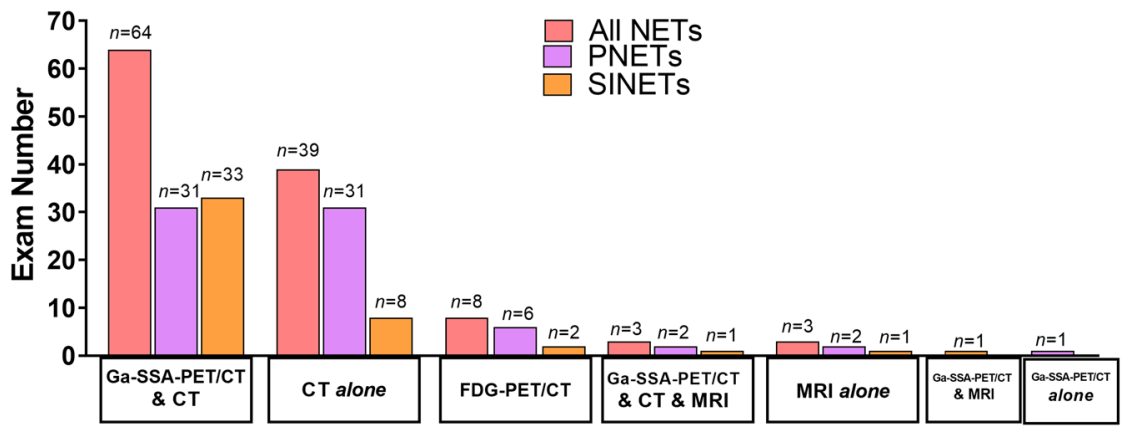

Figure 1

Summary of imaging modalities utilized in NETs, PNETs and SINETs. Anatomical imaging was performed in 110 NETs; functional: ${ }^{68} \mathrm{Ga}-\mathrm{SSA}$-PET/CT in 69 and ${ }^{18} \mathrm{~F}-\mathrm{FDG}-\mathrm{PET} / \mathrm{CT}$ in 8. Dual modality imaging: ${ }^{68} \mathrm{Ga}-\mathrm{SSA}-\mathrm{PET} / \mathrm{CT}$ and CT/MRI was performed in 68 NETs. All NETs: red; PNETs: purple; SINETs: orange. https://ec.bioscientifica.com https://doi.org/10.1530/EC-19-0030 (c) 2019 The authors Published by Bioscientifica Ltd
This work is licensed under a Creative Commons Attribution-NonCommercial-NoDerivatives 4.0 Internationab License.ifica com at $04 / 26 / 2023$ 11:44:54AM 
Table 1 Structure and clinicopathological data on the study cohort.

\begin{tabular}{|c|c|c|c|}
\hline Variables & PNET & SINET & Controls \\
\hline Number & 67 & 44 & 63 \\
\hline \multicolumn{4}{|l|}{ Gender } \\
\hline Males & 24 & 26 & 20 \\
\hline Females & 43 & 18 & 43 \\
\hline \multicolumn{4}{|l|}{ Age } \\
\hline Mean (range) & $56(19-87)$ & $59(27-77)$ & $44(23-78)$ \\
\hline \multicolumn{4}{|l|}{ Functionality status } \\
\hline Non-functional & 58 & 37 & N/A \\
\hline Functional & 9 & 7 & \\
\hline \multicolumn{4}{|l|}{ Grade } \\
\hline Grade 1 & 33 & 33 & $\mathrm{~N} / \mathrm{A}$ \\
\hline Grade 2 & 27 & 10 & \\
\hline Grade 3 NET & 2 & 1 & \\
\hline Grade 3 NEC & 3 & - & \\
\hline No data & 2 & - & \\
\hline \multicolumn{4}{|l|}{ TNM stage } \\
\hline Stage I & 21 & 2 & N/A \\
\hline Stage IIA & 8 & - & \\
\hline Stage IIB & 4 & - & \\
\hline Stage IIIA & 1 & - & \\
\hline Stage IIIB & 10 & 16 & \\
\hline Stage IV & 23 & 26 & \\
\hline \multicolumn{4}{|l|}{ Image-positive disease } \\
\hline No. & 42 & 33 & \\
\hline Stage I & 10 & - & N/A \\
\hline Stage IIA & 1 & - & \\
\hline Stage IIIB & 8 & 8 & \\
\hline Stage IV & 23 & 25 & \\
\hline \multicolumn{4}{|c|}{ Image-negative (dual modality) } \\
\hline Number & 8 & 3 & $\mathrm{~N} / \mathrm{A}$ \\
\hline \multicolumn{4}{|l|}{ Disease status (RECIST 1.1) } \\
\hline Stable & 34 & 30 & $\mathrm{~N} / \mathrm{A}$ \\
\hline Progressive & 8 & 3 & \\
\hline \multicolumn{4}{|l|}{ Current treatment } \\
\hline SSA & 27 & 27 & N/A \\
\hline Everolimus & 1 & 3 & \\
\hline Sunitinib & 1 & - & \\
\hline \multicolumn{4}{|l|}{ Previous treatments } \\
\hline \multirow[t]{6}{*}{ Primary tumor resection } & 44 & 39 & N/A \\
\hline & Distal pancreatectomy: 32 & RHC: 25 & \\
\hline & PancD (Traverso): 6 & SBR: 14 & \\
\hline & PancD (Whipple): 2 & - & \\
\hline & Enucleation: 3 & - & \\
\hline & Partial pancreatectomy: 1 & - & \\
\hline PRRT & 11 & 12 & \\
\hline Chemotherapy & 6 & 2 & \\
\hline Everolimus & 1 & $\mathrm{~N} / \mathrm{A}$ & \\
\hline Loco-regional (liver) & 6 & 3 & \\
\hline Radiotherapy & 3 & N/A & \\
\hline
\end{tabular}

Chemotherapy, FOLFOX, or paclitaxel + cisplatin, or etoposide + cisplatin; loco-regional therapy for liver metastases, liver resection, or RFA (radiofrequency ablation), or thermal ablation; PancD, pancreaticoduodenectomy; PRRT, peptide radioisotope receptor therapy; RHC, right hemicolectomy; SBR, small bowel resection; SSA, somatostatin analog.

The majority of SINETs (37/44) were NF, seven had CS. All were WD. Grade: G1: 33, G2: 10, G3: 1 (Table 1).

At the time of blood draw: 29 PNETs were treated - SSA $(n=27)$, everolimus $(n=1)$, sunitinib $(n=1)$; a majority had undergone other treatments: pancreatic resection $(n=44)$, chemotherapy $(n=6)$, everolimus $(n=1)$, PRRT $(n=11)$, loco-regional treatment for liver metastases $(n=6)$, radiotherapy $(n=3)$. At blood draw, 30 SINETs were treated: SSA $(n=27)$, everolimus $(n=3)$; a majority had undergone other treatments: https://ec.bioscientifica.com https://doi.org/10.1530/EC-19-0030 (c) 2019 The authors Published by Bioscientifica Ltd
This work is licensed under a Creative Commons Attribution-NonCommercial-NoDerivatives 4.0 elnternationad ticense ifica . com at 04/26/2023 11:44:54AM 
bowel resection $(n=39)$, chemotherapy $(n=2)$, PRRT $(n=12)$, loco-regional treatment for liver metastases $(n=3)$ (Table 1$)$.

\section{Blood samples collection for NETest measurement}

Peripheral blood samples $(3 \mathrm{~mL})$ were collected in EDTA tubes, mixed and stored on ice. Tubes were anonymously coded and stored at $-80^{\circ} \mathrm{C}$ within $2 \mathrm{~h}$ of collection per standard molecular diagnostics protocols for PCR-based studies (24). Randomly selected coded blood samples were sent de-identified to Wren Laboratories (Branford, CT, USA).

\section{NETest measurement}

Details of the PCR methodology, mathematical analysis and validation have been published in detail, comprising a two-step protocol (RNA isolation/cDNA production and qPCR) from EDTA-collected whole blood (24, $25,26)$. The assay was undertaken in a USA clinically certified laboratory (Wren Laboratories CL-0704, CLIA 07D2081388). Transcripts (mRNA) were isolated from EDTA-collected whole blood samples (mini blood kit, Qiagen) and real-time PCR was performed on prespotted plates. Target transcript levels are normalized and quantified versus a population control $(24,25,26)$. Final results are expressed as an activity index (NETest score) from 0 to $100 \%(24,25,26)$. Normal score cut-off: 20 .

\section{Statistical analysis}

The required total sample size (NETs and controls, power 0.8 and $\alpha=0.05$ ) to attain significant differences in NETest scores was calculated to be a minimum of 42 patients/subjects in each group. Intergroup analyses were undertaken using two-tailed non-parametric tests (Mann-Whitney $U$ test). AUROC analysis was used to determine the diagnostic accuracy of the NETest $(27,28,29)$. Metrics calculated included sensitivity and specificity. Prism 7.0 for Windows (GraphPad Software, www.graphpad.com) and MedCalc Statistical Software version 16.2.1 (MedCalc Software bvba, Ostend, Belgium; http://www.medcalc.org; 2017) were utilized. Statistical significance was defined as a $P$ value $<0.05$. Data are presented as mean \pm SD.

\section{Results}

\section{Disease extent evaluation}

Overall, IPD (any modality) was present in 75: PNETs $(n=42)$ - primary in situ/local recurrence $(n=26)$, lymph node metastases $(n=21)$, distant metastases (liver and/or bone, other: $n=23)$, SINETs $(n=33)$ : primary in situ $(n=4)$, lymph node metastases $(n=25)$, distant metastases (liver and/or bone, other: $n=26$ ) (Table 1 ). IND was confirmed by dual (both anatomical and functional negative) imaging in 11 WD NETs (PNET: $n=8$; SINET: $n=3$ ). These were considered 'true' negative by imaging. Disease positive on imaging but with discordant results between anatomical and functional imaging was present in six NETs: one PNET (CT positive and ${ }^{68} \mathrm{Ga}$-SSA-PET/CT negative) and five SINETs (all CT negative and ${ }^{68} \mathrm{Ga}-\mathrm{SSA}-\mathrm{PET} / \mathrm{CT}$ positive).

\section{Diagnosis}

NETest levels were significantly increased $(26 \pm 21)$ in all GEP-NETs $(n=111)$ versus controls $(8 \pm 4, P<0.0001)$ (Fig. 2). NETest diagnostic metrics were accuracy (97\%), sensitivity (99\%) and specificity (95\%). In IPD, NETest levels were $36 \pm 22$ and in IND significantly lower $(8 \pm 7$, $P<0.0001)$. NETest levels in image-positive PNETs $(37 \pm 22)$ and SINETs $(34 \pm 21)$ were not different. The AUROC for differentiating all NETs (irrespective of imaging status) from controls was 0.89 (95\% CI: 0.830-0.930, $P<0.0001)$. The AUROC for differentiating IPD from IND was 0.96 (95\% CI: 0.901-0.993, $P<0.0001)$ (Fig. 3). In IPD, NETest levels were not significantly different between TNM stages or grades (Fig. 4). Eight PNETs (one stage I, seven stage IV) and three SINETs (all stage IV) were progressive by imaging (RECIST). Among these, seven NETs (four PNETs, three SINETs, all stage IV) had a high NETest score $(79 \pm 10$; range 67-93\%).

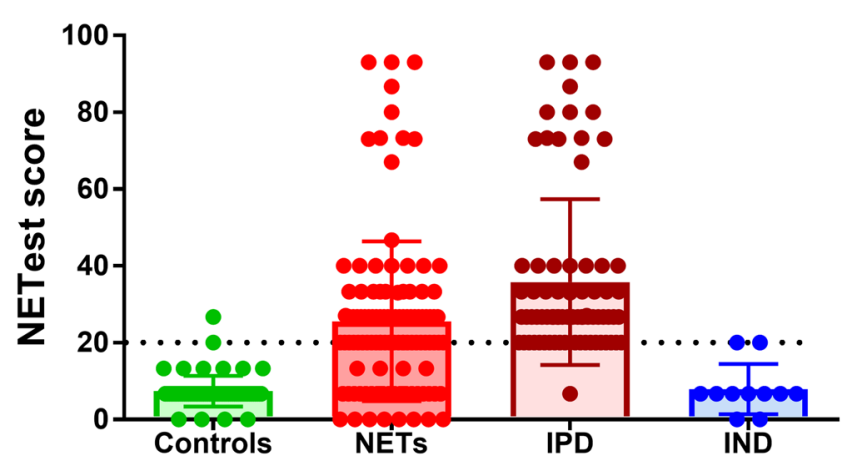

Figure 2

NETest in all NETs (SINETs and PNETs) and controls. Comparison of image-negative (IND) and image-positive NETs (IPD). The NETest measurements were elevated in the NET cohort $(26 \pm 21 ; n=111)$ compared to controls $(8 \pm 4, P<0.0001 ; n=63)$. NETest levels were elevated $(36 \pm 22)$ in IPD $(n=75)$ compared to IND $(n=11)(8 \pm 7$, $P<0.0001$ ). Two-tailed non-parametric tests (Mann-Whitney $U$ test). Statistical significance was defined at a $P$ value $<0.05$. Mean \pm SD.
This work is licensed under a Creative Commons Attribution-NonCommercial-NoDerivatives 4.0 internationab ticense.ifica.com at 04/26/2023 11:44:54AM 
A

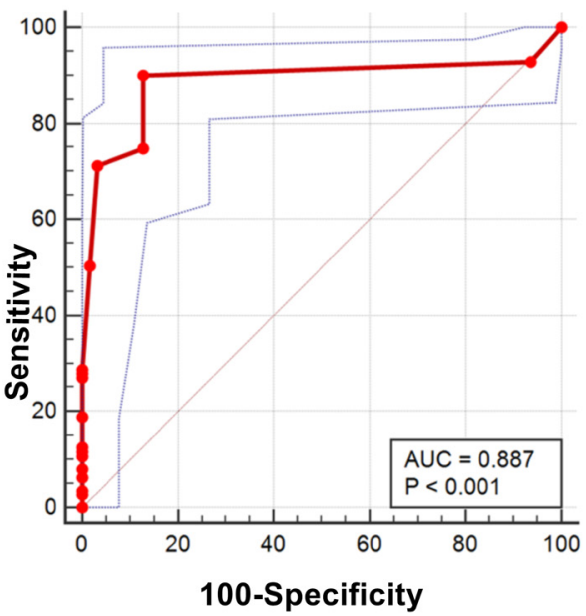

Concordance with imaging

\section{PNETS}

CT or MRI was performed in 66, 42 were positive and 24 negative. All 42 had a positive NETest (100\%). Twentyone of the 24 had a negative NETest. ${ }^{68} \mathrm{Ga}$-SSA-PET/CT was performed in 34; 24 were positive and all 24 had a positive NETest. Eight of the ten who were negative by ${ }^{68} \mathrm{Ga}$-SSA-PET/CT also had a negative NETest. Dual modality (CT or MRI and ${ }^{68} \mathrm{Ga}$-SSA-PET/CT) was available in 33, 25 were image positive and all had an elevated NETest. Among eight INDs, seven had a negative NETest (Fig. 5 and Table 2).

\section{SINETS}

CT or MRI was performed in 44, 28 were positive and 16 negative. All 28 image positive had a positive NETest. Ten of the 16 image negative had a negative NETest. ${ }^{68} \mathrm{Ga}$-SSA-PET/CT was performed in $35 ; 32$ were positive and the majority (31/32) had a positive NETest. Two of the three who were negative by imaging also had a negative NETest. Dual modality was available in 35, 32 were image positive and 31 had elevated NETest. Among three IND, two had a negative NETest.

Overall, the NETest was 92\% (101/110) concordant with anatomical imaging (CT or MRI), 94\% (65/69) concordant with ${ }^{68} \mathrm{Ga}$-SSA-PET/CT and 96\% (65/68) concordant with dual modality imaging (CT/MRI and ${ }^{68} \mathrm{Ga}-\mathrm{SSA}-\mathrm{PET} / \mathrm{CT}$ ) (Fig. 5 and Table 2). In image-positive CT/MRI $(n=70)$, NETest was elevated in all $70(37 \pm 22)$. In CT/MRI-negative $(n=40)$, NETest was normal $(11 \pm 10)$ in 31 . In image-positive ${ }^{68} \mathrm{Ga}-\mathrm{SSA}-\mathrm{PET} / \mathrm{CT}(n=56)$, the NETest was elevated $(36 \pm 22)$ in 55. In image-negative ${ }^{68} \mathrm{Ga}-S S A-P E T / C T ~(n=13)$, the NETest was normal in $10(9 \pm 8)$.

\section{Figure 3}

(A) The AUROC for NETest levels in NETs and controls: The AUROC for differentiating NETs from controls was 0.89 (95\% Cl: $0.830-0.930, P<0.0001)$. Statistical significance was defined at a $P$ value $<0.05$. (B) The AUROC for image-positive NETs (IPD) and image-negative (IND) NETs: The AUROC for differentiating IPD from subjects who were both anatomical and functional imaging negative for disease was 0.965 (95\% Cl: 0.901-0.993, $P<0.0001)$. Statistical significance was defined at a $P$ value $<0.05$

There were six image-discordant results (all in WD NETs): five ${ }^{68} \mathrm{Ga}-S S A-P E T / C T$ positive/CT negative with NETest positive (Fig. 6) in four and one ${ }^{68} \mathrm{Ga}$-SSA-PET/CT-negative/CT-positive with NETest positive. Among dual modality positive $(n=57), 56$ had elevated NETest. Nine of 11 INDs had a NETest within normal range $(8 \pm 7)$.

\section{Disease status}

Disease status assessed by RECIST 1.1: progressive disease $(n=11$; PNET, $n=8 ;$ SINET, $n=3)$. Stable disease $(n=64$; PNET: $n=34$; SINET: $n=30)$. PD NETest levels were significantly $(P<0.0001)$ higher $(61 \pm 26)$ than in stable disease (SD) (29 \pm 14$)$ (Fig. 7A). The majority (61/64) of NETs adjudged image stable (Fig. 7B) had a low NETest. Three exhibited a high score consistent with biochemical evidence of clinical disease activity or progression. Seven (7/11) individuals adjudged to be image progressive had a high score $(79 \pm 10)$. All seven were identified as having multiple, large $(>10 \mathrm{~mm})$ new lesions. In four with lower scores $(44 \pm 6)$, progression was considered to have occurred based upon the identification of a single new $<10 \mathrm{~mm}$ lesion over a median 12 months. The overall accuracy of the NETest for differentiating PD from SD based on a cut-off of NETest of $40(23,24,26)$ was $95 \%$.

\section{Discussion}

There has been considerable discussion about the need for an effective biomarker with clinical utility in NETs. Consensus statements have concluded that current monoanalyte biomarkers have diminished clinical utility (30). The previous default biomarker

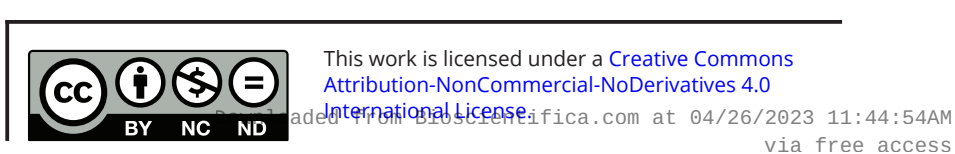


A

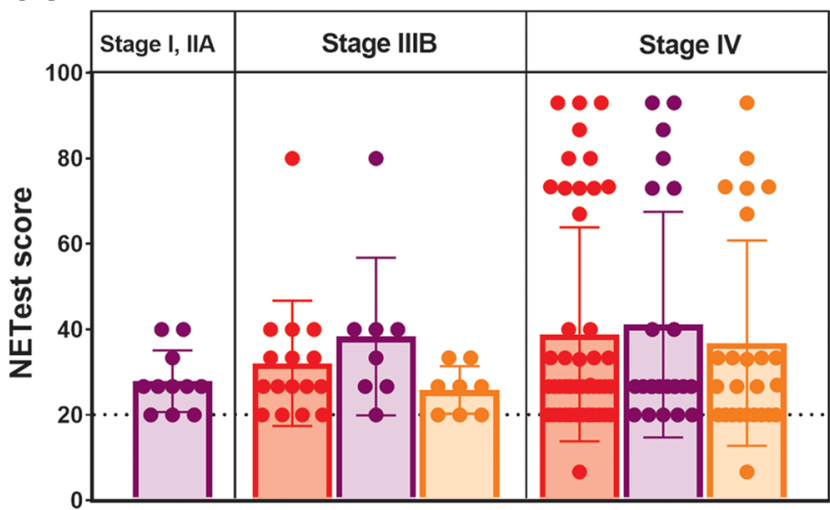

B

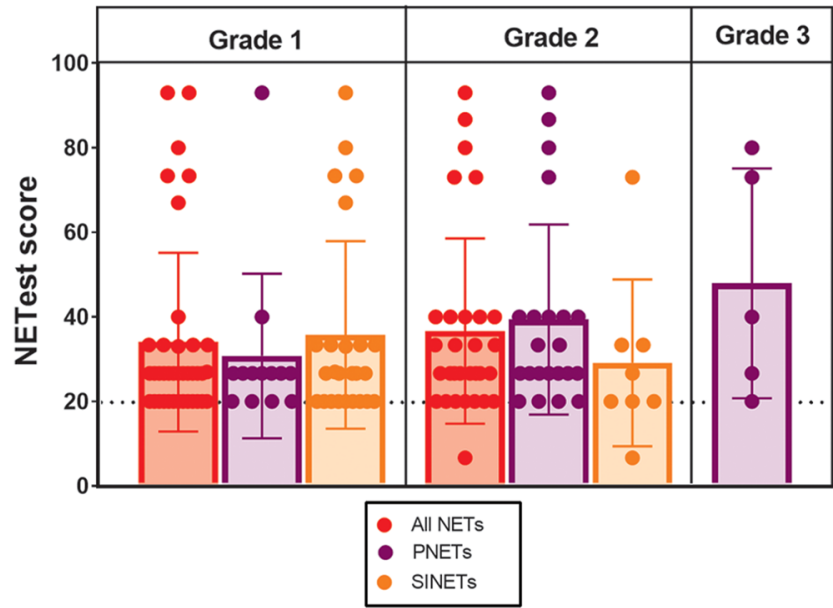

Figure 4

NETest levels in IPD according to TNM stage or grade. (A) NETest in IPD according to TNM stage $(5,36,37)$ : NETest levels were not significantly different between TNM stages; NETest in localized disease (PNETs: stage and IIA, $n=11$ ) was $28 \pm 7$; in stage IIIB NETs $(n=16)$ : $32 \pm 15$, PNETs $(n=8)$ $38 \pm 18$, SINETs $(n=8): 26 \pm 6$; in stage IV NETs $(n=48): 39 \pm 25$, PNETs $(n=23): 41 \pm 26$, SINETs ( $n=25): 37 \pm 24$ and were not significantly different. Two-tailed non-parametric tests (Mann-Whitney $U$ test). Statistical significance was defined at a $P$ value $<0.05$. Mean \pm SD. (B) NETest in IPD according to grade: NETest levels were not significantly different between grades; NETest in G1: NETs $(n=38)$ : $34 \pm 21$, PNETs $(n=13): 31 \pm 19$, SINETs $(n=25): 36 \pm 22$; in G2 NETs $(n=30): 37 \pm 21$, PNETs $(n=22): 39 \pm 22$, SINETs $(n=8): 29 \pm 20$; in G3 PNETs $(n=5): 48 \pm 27$ and were not significantly different. Two-tailed non-parametric tests (MannWhitney $U$ test). Statistical significance was defined at a $P$ value $<0.05$. Mean \pm SD

CgA is known to have limited if any clinical value in discriminant analysis studies (30). In the current study, the NETest was 97\% accurate, 99\% sensitive and $95 \%$ specific for a NET diagnosis. These metrics meet the NIH-proposed criteria of an optimal diagnostic biomarker (30) and are concordant with data from the previously reported GEP-NET studies. In a prospective study, NETest was $93 \%$ accurate in

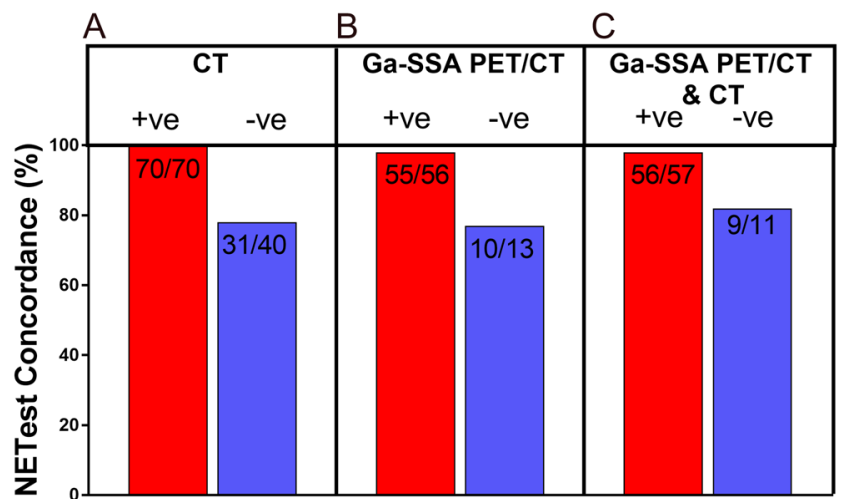

\section{Figure 5}

NETest concordance with imaging (morphologic and functional). Positive $=$ red. Negative $=$ blue. (A) Anatomical imaging was performed in 110 NETs (CT in 103, MRI in 4, CT and MRI in 3): 70 image positive, with NETest elevated in all; 40 image negative, with NETest normal in 31, and NETest elevated in 9: 4 were ${ }^{68} \mathrm{Ga}$-SSA-PET/CT positive; 3 had only single imaging modality performed ( $C T$ in $2, M R I$ in 1 ) and 2 were dual modality negative. (B) ${ }^{68} \mathrm{Ga}-\mathrm{SSA}-\mathrm{PET} / \mathrm{CT}$ was performed in $69:{ }^{68} \mathrm{Ga}-\mathrm{SSA}-\mathrm{PET} / \mathrm{CT}$ was positive in 56 , with NETest elevated in $55 ;{ }^{68} \mathrm{Ga}-\mathrm{SSA}$-PET/CT was negative in 13 with NETest normal in 10. (C) Dual modality imaging was performed in 68. Image positive $(n=57)$, NETest was elevated in 56; 11 were dual image negative with NETest normal in 9.

identifying SINET and 94\% for PNET diagnosis (31). In a large cohort ( $n=140$ NETs, $n=113$ controls) from the Netherlands, the diagnostic accuracy was 93\% (32). In a separate real-world study of 100 NET patients undertaken in US secondary and tertiary institutions, the diagnostic accuracy was confirmed as $97 \%$ in GEP-NETs (23).

To assess the NETest diagnostic efficacy, we compared its concordance with radiological evidence of the disease, since imaging is the critical arbiter in NET disease diagnostic work-up and monitoring (9). The concordance between a positive NETest score and imaging was high (>90\%). Specifically, it was 92\% (101/110) with anatomical imaging, 94\% (65/69) with functional imaging and 96\% (65/68) when compared to two imaging modalities (either CT or MRI and ${ }^{68} \mathrm{Ga}$-SSA-PET/CT) were combined. In IPD, the NETest was 70/70 concordant with anatomical imaging, and it was 55/56 concordant with ${ }^{68} \mathrm{Ga}$-SSA-PET/CT. In one case, the NETest was normal and CT was negative, while ${ }^{68} \mathrm{Ga}$-SSA-PET/CT showed increased tracer uptake in three abdominal lymph nodes and focal uptake in the liver segment II. This patient developed liver metastases detectable by anatomical imaging (CT) 7 months later.

In the assessment of NETest concordance with imagenegative NETs, there was a 'lower' concordance between the NETest and CT-negative results (31/40). This was, for the most part, due to false-negative CT results. In five cases

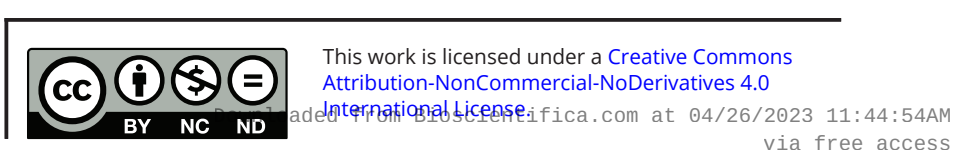


Table 2 Summary of the NETest concordance with imaging performed in the entire NET cohort.

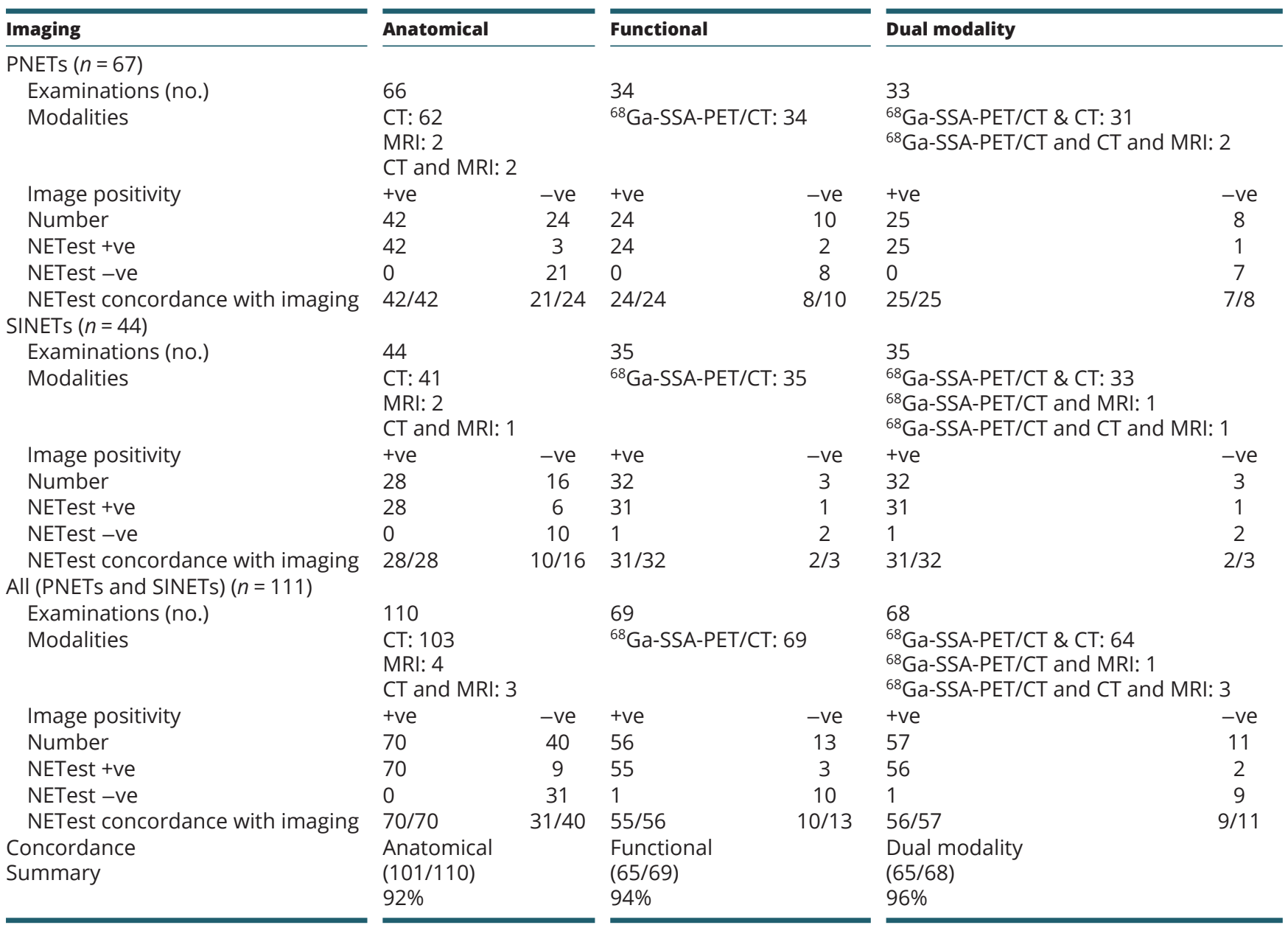
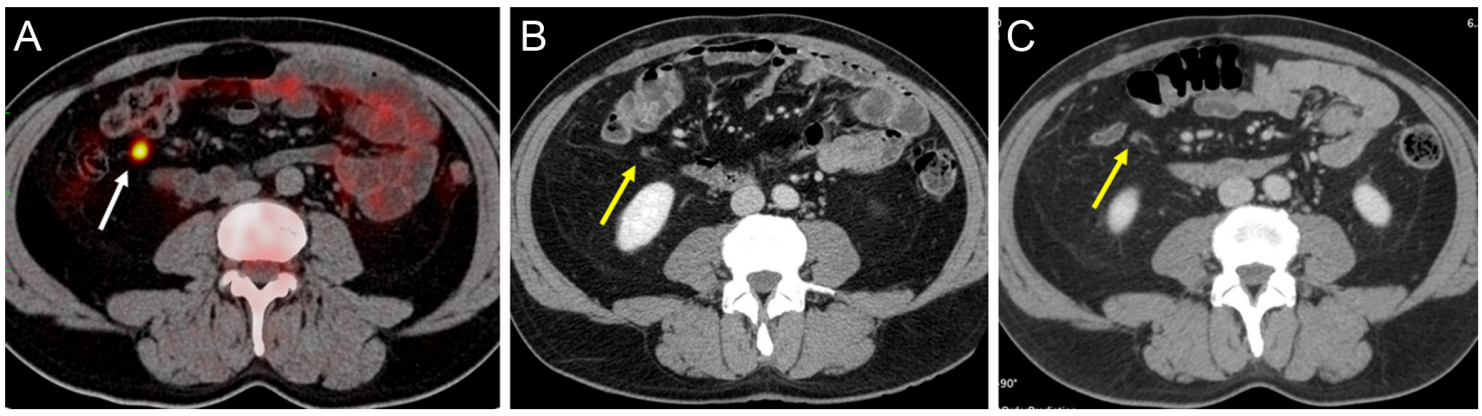

\section{Figure 6}

Elevated NETest measurement correlates with ${ }^{68} \mathrm{Ga}$-DOTA-TATE PE/CT in identifying metastatic disease, while CT remains negative. A patient diagnosed with SINET G1, 7 years post resection (p[m]T3NOMO, RO, LVI 0, Ki-67 1\%) exhibited a positive NETest measurement (27\%). (A) ${ }^{68}$ Ga-DOTA-TATE PET/CT identified two tracer-avid subcentimeter mesenteric nodes/nodules (SUVmax 13.3 - white arrow and 8.7 - adjacent to the right aspect of the transverse colon). (B) Diagnostic abdominal CT was obtained which confirmed the absence of pathologically enlarged nodes/nodules at the site of the ${ }^{68} \mathrm{Ga}-\mathrm{SSA}$ PET/ CT uptake (yellow arrow). The patient subsequently developed abdominal pain and diarrhea and SSA therapy was initiated. (C) A follow-up diagnostic abdominal CT 16 months later again did not identify any pathologically enlarged node at this site (yellow arrow). LVI, lymphovascular invasion; R, resection margin; SSA, somatostatin analog. 


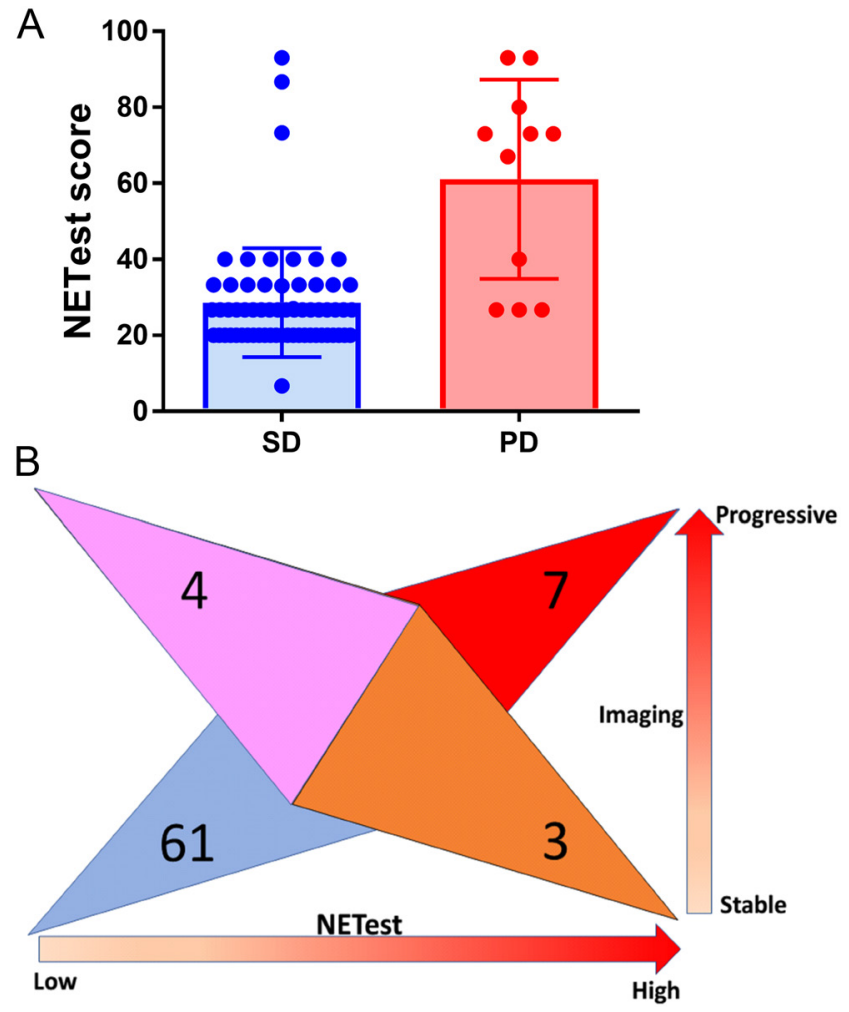

Figure 7

NETest levels in stable and progressive disease. (A) NETest in stable vs progressive disease: NETest was significantly elevated in PD $(n=11)$ $(61 \pm 26)$ compared to stable disease $(n=64)(29 \pm 14)(P<0.0001)$. Two-tailed non-parametric tests (Mann-Whitney $U$ test). Statistical significance was defined at a $P$ value $<0.05$. Mean \pm SD. (B) Concordance of image- and NETest-based disease progression: The majority (95\%) of patients $(n=61)$ who were considered image stable (bottom left) had a low NETest. Three exhibited a high score consistent with biochemical evidence of disease progression. Sixty-four percent $(n=7)$ of imageprogression patients had a high score which correlated with the identification of multiple new large lesions at imaging. A further four patients had low scores; all of whom were identified with single, new small solitary lesion over a median 12-month follow-up.

with both CT and ${ }^{68} \mathrm{Ga}-\mathrm{SSA}$-PET/CT imaging available, CT was negative but ${ }^{68} \mathrm{Ga}$-SSA-PET/CT identified lymph node metastases (in three), and in two others, lymph node as well as liver metastases were evident. In four, the NETest was positive. The NETest therefore identifies lesions that are not detected by CT.

Based on these observations as well as the known sensitivity discrepancy between morphologic and functional $\left({ }^{68} \mathrm{Ga}\right.$-SSA-PET/CT) imaging modalities, we considered a patient image negative when confirmed by two modalities (CT or MRI and ${ }^{68} \mathrm{Ga}-S S A-P E T / C T$ in WD NETs). A double image-negative status demonstrated a clearer indication of concordance between the NETest assay and those without disease. There were 11 NETs (8 PNETs, 3 SINETs) with dual modality imaging and no radiological evidence of disease at blood draw. In two, the NETest was positive, but in each case, the results were borderline positive (20\%). In one of these, a blood draw took place 5 months after distal pancreatectomy for stage I NF-PNET (resection margin-negative (R0); Ki-67 1\%). In the other, a SINET, blood was collected 1 month after right hemicolectomy for an ileocecal valve NET (pT3N1, R0; Ki-67 1\%); 12 months later, a follow-up CT was negative. It is likely in these two cases that the NETest was detecting microscopic disease.

Among 24 CT-negative or MRI-negative cases (in which ${ }^{68} \mathrm{Ga}$-SSA-PET/CT was not available at the time of blood draw), three cases had moderately elevated NETest: two PNETs (CT negative; NETest scores: 33\% and 47\%) and one SINET (MRI negative; NETest score: 27\%). This was indicative of NET disease but with low biological activity. The first case (NF-PNET, Ki-67 4\%; 15 months after distal pancreatectomy, pT4N0), at the time of a blood draw, had two hyper-vascular lesions (segment VIII) identified by CT. On a follow-up MRI, these were defined as perfusion disturbances; a ${ }^{68} \mathrm{Ga}$-SSA-PET/CT performed thereafter (within 4 months) revealed slightly increased tracer uptake in orbital bones, without any corresponding lesions on a CT, and with no pathological uptake in the liver - further radiological follow-up was recommended. Another case (NETest 47\%), NF-PNET $~ 3.8$ years after pancreaticoduodenectomy (Traverso, pT3NO, Ki-67 $<2 \%$ ), at the time of blood draw was CT negative (there were unsuspicious liver cysts). A follow-up ${ }^{68} \mathrm{Ga}$-SSA-PET/CT 3 months post blood draw was normal. The third case (SINET), 5 years after right hemicolectomy for ileocecal valve NET (stage I, R0, Ki-67 <1\%), was MRI negative at blood draw. Six months earlier, the ${ }^{68} \mathrm{Ga}$-SSA-PET/CT was negative. It is likely that these results represent the fact that elevations in NETest can precede alterations in imaging by (6-24 months). Previous reports have documented that elevations in the NETest are consistent with disease progression and are detectable in blood a significant time period before image alterations are evident $(24,26)$. For example, in a surgical series, those with microscopic disease (positive resection margins) and an elevated NETest (despite negative imaging at the time of blood draw) developed surgical recurrence within 6-12 months after R0 resection $(25,33)$.

There were six NET subjects in whom the morphologic and functional imaging results were discordant: five ${ }^{68} \mathrm{Ga}$-SSA-PET/CT positive and CT negative with NETest positive in four and one ${ }^{68} \mathrm{Ga}$-SSA-PET/CT negative but CT positive which was NETest positive. In cases where CT was negative, while ${ }^{68} \mathrm{Ga}-S S A-P E T / C T$ was positive, 
the 'lower' concordance of the NETest with CT reflects 'falsenegative' results in this imaging modality are consistent with the known increased efficacy of functional imaging $(13,15) .{ }^{68} \mathrm{Ga}$-SSA-PET/CT is considered more sensitive than CT in the detection of WD lesions (13), with Ki-67 $<15 \%$; however, in one patient, the CT was positive (single small liver lesion) and ${ }^{68} \mathrm{Ga}$-SSA-PET/CT was negative (a well-known limitation of SRS; Ki-67 3\%).

Tumor heterogeneity has an impact on imaging sensitivity; therefore, it is apparent that no single imaging modality can be exclusively used to diagnose or detect the disease. Overall, combined imaging strategies are more effective, if available. However, follow-up with multiple imaging tools has risks (increased radiation exposure) and represents a high economic burden for the healthcare system. Moreover, resolution limitations (and the consequent difficulties with detection of meaningful size changes, especially in slow-growing or high-burden disease) result in the need for repetitive imaging, for example, every 3 months. Overall, imaging, especially when used to apply RECIST, has limitations. Thus, RECIST is inadequate for the early assessment of progression. This is a particular problem in NET disease, where the disease tends to grow slowly and, by definition, no progression is evident until the sum of the longest diameters has increased by $20 \%$. In the case of volume, this therefore requires a substantial increase in tumor volume before it is defined as progression. Alternatively, for smallvolume disease $(2-3 \mathrm{~mm})$, this $20 \%$ value cannot be easily identified and leads to incorrect attribution of progression in case of human-based measurement errors. The wellestablished RECIST 1.1 considers only a maximum of five lesions in total, maximum of two per organ. Certainly, in NET disease, where patients frequently present with extensive metastases, a sum of five lesions may well be non-representative of the entirety of the disease. In addition, bone disease is non-measurable, by definition, since the majority of bone metastases are (faintly) sclerotic and not lytic and frequently seen on ${ }^{68} \mathrm{Ga}$-DOTA-TATE PET/CT, rather than CT or MRI, which are the techniques considered in RECIST. RECIST 1.1 also includes PET (FDG-PET for solid tumors) to complement CT in the disease assessment. In NETs, FDG-PET is notoriously inefficient to detect the entirety of the disease and, to date, ${ }^{68} \mathrm{Ga}$-DOTATATE and/or a combination of FDG- and ${ }^{68} \mathrm{Ga}$-DOTA-TATE-PET/CT has not been incorporated in the response evaluation criteria. It has also been recognized that molecular imaging responses can also precede the morphologic response by several weeks (34).
Given the limitations of imaging, the use of an adjunctive strategy such as liquid biopsy may provide important information. Thus, a non-invasive liquid biopsy such as the NETest, which functions as an accurate diagnostic, and as noted in this study, correlates with imaging, is of likely clinical value.

It has been reported that the 'omic' cluster analysis which forms the basis of the multianalyte algorithm of the NETest are accurate in differentiating stable from progressive disease (35). In our cohort, 11 patients (8 PNET, 3 SINET) exhibited progressive and 64 stable disease. NETest levels were significantly elevated in PD $(61 \pm 26)$ compared to SD $(29 \pm 14)$. Using a cut-off of 40 $(23,24,26)$, the NETest was $95 \%$ accurate in differentiating SD from PD. The identification of multiple, large $(>10 \mathrm{~mm})$ new lesions were associated with high scores $(79 \pm 10)$. Single, new smaller lesions were associated with lower scores $(44 \pm 6)$. Three patients of those considered SD $(3 / 64)$ had NETest scores $>40$. In one, clinical evidence of disease progression was noted at the time of blood draw. In the second, a subsequent CT 6 months after blood draw confirmed progression (target lesions $>20 \%$ ). The third patient (NF-PNET, Ki67 8\%) has previously undergone six cycles of PRRT. It is likely, given that imaging underestimates disease progression, that longer follow-up in this patient will demonstrate evidence of progression.

The majority of the image-positive cohort (54/75) was on SSA; therefore, the NETest can provide adjunctive information that supports the demonstration of therapeutic efficacy of SSA. The NETest utility in predicting SSA treatment response has already been reported in a separate NET cohort $(n=28)$ receiving SSAs (26). Multiple regression analysis in this cohort demonstrated that the NETest effectively predicted the onset of progressive disease while on SSA $(p=0.0002)$. Of more importance, NETest increased significantly earlier $(\sim 5$ months prior) than imaging for RECIST-defined disease progression (treatment failure) in this cohort. The assay was considered effective in the identification of progressive disease (26).

This clinical utility of the NETest has been recently reported in a real-world study based upon an independent registry (23). This study demonstrated a very good concordance (83-88\%) between the NETest with imaging, especially functional. It also provided useful preliminary information indicating that the NETest was effective in differentiating SD from progression and that it may be more sensitive in detecting progression before it is apparent on imaging. A prospective, large study is needed 
to precisely define how effective the NETest alone is in identifying progression and how much earlier it can detect alterations in disease status.

It should be noted that the current study was based upon real-world principles. Therefore, although the entire study cohort comprised 174 subjects: 111 NET patients and 63 controls, the number of subjects in PNET and SINET subgroups was limited (reflecting a 12-month enrolment by one-center in a relatively rare disease). The follow-up period may have varied among the study subjects (median 8 months (1.5-24 months)). Furthermore, functional imaging was not available at blood draw in all $\left({ }^{68} \mathrm{Ga}\right.$-SSA-PET/CT was available in 69 and FDG-PET/CT in 8), and there were only 11 progressive cases while imaging. Since only six subjects were high grade (three well- and three poorly differentiated), this did not allow a sub-analysis of the NETest efficacy in this more aggressive group of neuroendocrine neoplasia. Based upon these limitations, we would propose that further assessment of the relationship of NETest and imaging would require a future, larger and prospective study to extend the current results. Nevertheless, despite these limitations, the current data provide clear evidence that a blood-based multigene biomarker provides accurate information that is concordant with imaging. Use of the NETest information obtained by venipuncture may provide a point of care basis for monitoring disease, thereby decreasing the health costs of imaging and patient exposure to radiation.

\section{Conclusions}

The NETest has been independently validated as an accurate diagnostic biomarker for SINET and PNET. These results are concordant with imaging and provide a good assessment of disease status. The NETest was as accurate as imaging as a diagnostic. The use of a multianalyte gene blood test and imaging may provide adjunctive information that can facilitate the management of NET disease.

\section{Declaration of interest}

The authors declare that there is no conflict of interest that could be perceived as prejudicing the impartiality of the research reported.

\section{Funding}

This work was supported by the Medical University of Silesia. Wren Laboratories provided sample measurement at no cost.

\section{References}

1 Dasari A, Shen C, Halperin D, Zhao B, Zhou S, Xu Y, Shih T \& Yao JC. Trends in the incidence, prevalence, and survival outcomes in patients With neuroendocrine tumors in the United States. JAMA Oncology 20173 1335-1342. (https://doi.org/10.1001/ jamaoncol.2017.0589)

2 Pavel M, O'Toole D, Costa F, Capdevila J, Gross D, Kianmanesh R, Krenning E, Knigge U, Salazar R, Pape UF, et al. Enets consensus guidelines update for the management of distant metastatic disease of intestinal, pancreatic, bronchial neuroendocrine neoplasms (NEN) and NEN of unknown primary site. Neuroendocrinology 2016103 172-185. (https://doi.org/10.1159/000443167)

3 Frilling A, Modlin IM, Kidd M, Russell C, Breitenstein S, Salem R, Kwekkeboom D, Lau WY, Klersy C, Vilgrain V, et al. Recommendations for management of patients with neuroendocrine liver metastases. Lancet Oncology 201415 e8-e21. (https://doi. org/10.1016/S1470-2045(13)70362-0)

4 Falconi M, Eriksson B, Kaltsas G, Bartsch DK, Capdevila J, Caplin M, Kos-Kudla B, Kwekkeboom D, Rindi G, Kloppel G, et al. Enets consensus guidelines update for the management of patients with functional pancreatic neuroendocrine tumors and non-functional pancreatic neuroendocrine tumors. Neuroendocrinology 2016103 153-171. (https://doi.org/10.1159/000443171)

5 Kos-Kudla B, Rosiek V, Borowska M, Baldys-Waligorska A, Bednarczuk T, Blicharz-Dorniak J, Bolanowski M, Boratyn-Nowicka A, Cichocki A, Cwikla JB, et al. Pancreatic neuroendocrine neoplasms - management guidelines (recommended by the Polish Network of Neuroendocrine Tumours). Endokrynologia Polska 201768 169-197. (https://doi.org/10.5603/EP.2017.2016)

6 Srirajaskanthan R, Shanmugabavan D \& Ramage JK. Carcinoid syndrome. BMJ 2010341 c3941. (https://doi.org/10.1136/bmj.c3941)

7 Ruf J, Schiefer J, Kropf S, Furth C, Ulrich G, Kosiek O, Denecke T, Pavel M, Pascher A, Wiedenmann B, et al. Quantification in (68) Ga-DOTA(0)-Phe(1)-Tyr(3)-octreotide positron emission tomography/ computed tomography: can we be impartial about partial volume effects? Neuroendocrinology 201397 369-374. (https://doi. org/10.1159/000350418)

8 Virgolini I, Ambrosini V, Bomanji JB, Baum RP, Fanti S, Gabriel M, Papathanasiou ND, Pepe G, Oyen W, De Cristoforo C, et al. Procedure guidelines for PET/CT tumour imaging with 68Ga-DOTA-conjugated peptides: 68Ga-DOTA-TOC, 68Ga-DOTA-NOC, 68Ga-DOTA-TATE. European Journal of Nuclear Medicine and Molecular Imaging 201037 2004-2010. (https://doi.org/10.1007/s00259-010-1512-3)

9 Oberg K, Krenning E, Sundin A, Bodei L, Kidd M, Tesselaar M, Ambrosini V, Baum RP, Kulke M, Pavel M, et al. A Delphic consensus assessment: imaging and biomarkers in gastroenteropancreatic neuroendocrine tumor disease management. Endocrine Connections 20165 174-187. (https://doi.org/10.1530/EC-16-0043)

10 Kidd M, Bodei L \& Modlin IM. Chromogranin A: any relevance in neuroendocrine tumors? Current Opinion in Endocrinology, Diabetes, and Obesity 201623 28-37. (https://doi.org/10.1097/ MED.0000000000000215)

11 de Mestier L, Dromain C, d'Assignies G, Scoazec JY, Lassau N, Lebtahi R, Brixi H, Mitry E, Guimbaud R, Courbon F, et al. Evaluating digestive neuroendocrine tumor progression and therapeutic responses in the era of targeted therapies: state of the art. EndocrineRelated Cancer 201421 R105-R120. (https://doi.org/10.1530/ERC-130365)

12 Schwartz LH, Litière S, de Vries E, Ford R, Gwyther S, Mandrekar S, Shankar L, Bogaerts J, Chen A, Dancey J, et al. RECIST 1.1-update and clarification: from the RECIST committee. European Journal of Cancer 201662 132-137. (https://doi.org/10.1016/j.ejca.2016.03.081)

13 Bodei L, Sundin A, Kidd M, Prasad V \& Modlin IM. The status of neuroendocrine tumor imaging: from darkness https://ec.bioscientifica.com https://doi.org/10.1530/EC-19-0030 (c) 2019 The authors Published by Bioscientifica Ltd
This work is licensed under a Creative Commons Attribution-NonCommercial-NoDerivatives 4.0 enternationad License.ifica com at $04 / 26 / 2023$ 11:44:54AM 
to light? Neuroendocrinology 2015101 1-17. (https://doi. org/10.1159/000367850)

14 Choi H, Charnsangavej C, Faria SC, Macapinlac HA, Burgess MA, Patel SR, Chen LL, Podoloff DA \& Benjamin RS. Correlation of computed tomography and positron emission tomography in patients with metastatic gastrointestinal stromal tumor treated at a single institution with imatinib mesylate: proposal of new computed tomography response criteria. Journal of Clinical Oncology 200725 1753-1759. (https://doi.org/10.1200/JCO.2006.07.3049)

15 Toumpanakis C, Kim MK, Rinke A, Bergestuen DS, Thirlwell C, Khan MS, Salazar R \& Oberg K. Combination of crosssectional and molecular imaging studies in the localization of gastroenteropancreatic neuroendocrine tumors. Neuroendocrinology 201499 63-74. (https://doi.org/10.1159/000358727)

16 Gibson WE, Gonzalez RS, Cates JMM, Liu E \& Shi C. Hepatic micrometastases are associated with poor prognosis in patients with liver metastases from neuroendocrine tumors of the digestive tract. Human Pathology 201879 109-115. (https://doi.org/10.1016/j. humpath.2018.05.006)

17 Malczewska A, Bodei L, Kidd M \& Modlin IM. Blood mRNA measurement (NETest) for neuroendocrine tumors diagnosis of imagenegative liver metastatic disease. Journal of Clinical Endocrinology and Metabolism 2019104 867-872. (https://doi.org/10.1210/jc.2018-01804)

18 Niederle B, Pape UF, Costa F, Gross D, Kelestimur F, Knigge U, Oberg K, Pavel M, Perren A, Toumpanakis C, et al. Enets consensus guidelines update for neuroendocrine neoplasms of the jejunum and ileum. Neuroendocrinology 2016103 125-138. (https://doi. org/10.1159/000443170)

19 Bodei L, Kidd MS, Singh A, van der Zwan WA, Severi S, Drozdov IA, Cwikla J, Baum RP, Kwekkeboom DJ, Paganelli G, et al. PRRT genomic signature in blood for prediction of (177)Lu-octreotate efficacy. European Journal of Nuclear Medicine and Molecular Imaging 201845 1155-1169. (https://doi.org/10.1007/s00259-018-3967-6)

20 Siravegna G, Marsoni S, Siena S \& Bardelli A. Integrating liquid biopsies into the management of cancer. Nature Reviews Clinical Oncology 201714 531-548. (https://doi.org/10.1038/ nrclinonc.2017.14)

21 Modlin IM, Drozdov I \& Kidd M. The identification of gut neuroendocrine tumor disease by multiple synchronous transcript analysis in blood. PLoS ONE 20138 e63364. (https://doi.org/10.1371/ journal.pone.0063364)

22 Modlin IM, Kidd M, Malczewska A, Drozdov I, Bodei L, Matar S \& Chung KM. The NETest: the clinical utility of multigene blood analysis in the diagnosis and management of neuroendocrine tumors. Endocrinology and Metabolism Clinics of North America 2018 47 485-504. (https://doi.org/10.1016/j.ecl.2018.05.002)

23 Liu E, Paulson S, Gulati A, Freudman J, Grosh W, Kafer S, Wickremesinghe PC, Salem RR \& Bodei L. Assessment of NETest clinical utility in a U.S. Registry-based study. Oncologist 2018231. (https://doi.org/10.1634/theoncologist.2017-0623)

24 Pavel M, Jann H, Prasad V, Drozdov I, Modlin IM \& Kidd M. NET blood transcript analysis defines the crossing of the clinical Rubicon: when stable disease becomes progressive. Neuroendocrinology 2017 104 170-182. (https://doi.org/10.1159/000446025)

25 Filosso PL, Kidd M, Roffinella M, Lewczuk A, Chung KM, KolasinskaCwikla A, Cwikla J, Lowczak A, Doboszynska A, Malczewska A, et al. The utility of blood neuroendocrine gene transcript measurement in the diagnosis of bronchopulmonary neuroendocrine tumours and as a tool to evaluate surgical resection and disease progression. European
Journal of Cardio-Thoracic Surgery 201853 631-639. (https://doi. org/10.1093/ejcts/ezx386)

26 Cwikla JB, Bodei L, Kolasinska-Cwikla A, Sankowski A, Modlin IM \& Kidd M. Circulating transcript analysis (NETest) in GEP-NETs treated With somatostatin analogs defines therapy. Journal of Clinical Endocrinology and Metabolism 2015100 E1437-E1445. (https://doi. org/10.1210/jc.2015-2792)

27 Modlin IM, Aslanian H, Bodei L, Drozdov I \& Kidd M. A PCR blood test outperforms chromogranin A in carcinoid detection and is unaffected by proton pump inhibitors. Endocrine Connections 20143 215-223. (https://doi.org/10.1530/EC-14-0100)

28 Zweig MH \& Campbell G. Receiver-operating characteristic (ROC) plots: a fundamental evaluation tool in clinical medicine. Clinical Chemistry 199339 561-577.

29 Hanley JA \& McNeil BJ. A method of comparing the areas under receiver operating characteristic curves derived from the same cases. Radiology 1983148 839-843. (https://doi.org/10.1148/ radiology.148.3.6878708)

30 Oberg K, Modlin IM, De Herder W, Pavel M, Klimstra D, Frilling A, Metz DC, Heaney A, Kwekkeboom D, Strosberg J, et al. Consensus on biomarkers for neuroendocrine tumour disease. Lancet Oncology 2015 16 e435-e446. (https://doi.org/10.1016/S1470-2045(15)00186-2)

31 Modlin IM, Kidd M, Bodei L, Drozdov I \& Aslanian H. The clinical utility of a novel blood-based multi-transcriptome assay for the diagnosis of neuroendocrine tumors of the gastrointestinal tract. American Journal of Gastroenterology 2015110 1223-1232. (https:// doi.org/10.1038/ajg.2015.160)

32 van Treijen MJC, Korse CM, van Leeuwaarde RS, Saveur LJ, Vriens MR, Verbeek WHM, Tesselaar MET \& Valk GD. Blood transcript profiling for the detection of neuroendocrine tumors: results of a large independent validation study. Frontiers in Endocrinology 20189 740. (https://doi.org/10.3389/ fendo.2018.00740)

33 Modlin IM, Frilling A, Salem RR, Alaimo D, Drymousis P, Wasan HS, Callahan S, Faiz O, Weng L, Teixeira N, et al. Blood measurement of neuroendocrine gene transcripts defines the effectiveness of operative resection and ablation strategies. Surgery 2016159 336-347. (https://doi.org/10.1016/j.surg.2015.06.056)

34 Haug AR, Auernhammer CJ, Wangler B, Schmidt GP, Uebleis C, Goke B, Cumming P, Bartenstein P, Tiling R \& Hacker M. 68Ga-DOTATATE PET/CT for the early prediction of response to somatostatin receptor-mediated radionuclide therapy in patients with well-differentiated neuroendocrine tumors. Journal of Nuclear Medicine 201051 1349-1356. (https://doi.org/10.2967/ jnumed.110.075002)

35 Kidd M, Drozdov I \& Modlin I. Blood and tissue neuroendocrine tumor gene cluster analysis correlate, define hallmarks and predict disease status. Endocrine-Related Cancer 201522 561-575. (https:// doi.org/10.1530/ERC-15-0092)

36 Bednarczuk T, Bolanowski M, Zemczak A, Baldys-Waligorska A, Blicharz-Dorniak J, Boratyn-Nowicka A, Borowska M, Cichocki A, Cwikla JB, Falconi M, et al. Neuroendocrine neoplasms of the small intestine and appendix - management guidelines (recommended by the Polish Network of Neuroendocrine Tumours). Endokrynologia Polska 201768 223-236. (https://doi. org/10.5603/EP.2017.0018)

37 Brierley JD, Gospodarowicz MK \& Wittekind Ch (eds). International Union Against Cancer (UICC). TNM Classification of Malignant Tumours, 8th ed. Oxford, UK: John Wiley \& Sons, 2017.

Received in final form 28 February 2019

Accepted 13 March 2019

Accepted Preprint published online 13 March 2019 https://ec.bioscientifica.com https://doi.org/10.1530/EC-19-0030 (c) 2019 The authors Published by Bioscientifica Ltd
This work is licensed under a Creative Commons Attribution-NonCommercial-NoDerivatives 4.0 Internationab sicense.ifica . com at 04/26/2023 11:44:54AM 